\title{
Saving mental effort to maintain physical effort: a shift of activity within the prefrontal cortex in anticipation of prolonged exercise
}

\author{
Rémi Radel $^{1,2} \cdot$ Jeanick Brisswalter $^{1} \cdot$ Stéphane Perrey $^{3}$
}

Published online: 17 November 2016

(C) Psychonomic Society, Inc. 2016

\begin{abstract}
Executive functioning and attention require mental effort. In line with the resource conservation principle, we hypothesized that mental effort would be saved when individuals expected to exercise for a long period. Twenty-two study participants exercised twice on a cycle ergometer for $10 \mathrm{~min}$ at $60 \%$ of their maximal aerobic power, with the expectation of exercising for either $10 \mathrm{~min}$ or $60 \mathrm{~min}$. Changes in activity in the right dorsolateral prefrontal cortex (rdlPFC) and right medial frontal cortex (rmPFC) were investigated by measuring oxyhemoglobin using near-infrared spectroscopy. Attentional focus and ratings of perceived exertion were assessed at three time points (200, 400, and $600 \mathrm{~s})$. The oxyhemoglobin concentration was lower in the rdIPFC and higher in the rmPFC under the 60-min than under the 10-min condition. Also, attention was less focused in the 60-min than in the 10-min condition. We discuss these results as possible evidence of a disengagement of the brain regions associated with mental effort (executive network), in favor of brain regions linked to resting activity (the default network), in order to save mental resources for the maintenance of exercise.
\end{abstract}

Keywords Hypofrontality $\cdot$ Physical exercise $\cdot$ Attentional focus $\cdot$ Executive function $\cdot$ Default network

Rémi Radel

remi.radel@unice.fr

1 Université Côte d'Azur, Laboratoire LAMHESS, Nice, France

2 Laboratoire LAMHESS (EA6309), Université de Nice Sophia-Antipolis, 261, route de Grenoble, 06205 Nice, cédex 3, France

3 EuroMov, University of Montpellier, Montpellier, France
Emerging evidence suggests that the maintenance of ongoing physical exercise requires mental effort. Mental effort is a feeling of strain occurring while investing a limited energetic resource to perform a mental task, due to the mobilization of controlled cognitive processing (Kahneman, 1973; Paas \& Van Merrienboer, 1993). Mental effort is thus required if a task requires top-down attention or inhibition of impulsive responses. For the maintenance of a physical task, theoretical models suggest that mental effort would be necessary to inhibit the bodily afferences that arise with physical fatigue (Audiffren \& André, 2015; Ekkekakis, 2009). In line with these models, the maintenance of ongoing physical exercise would mainly rely on inhibition. Since attentional focalization may not play a crucial role for exercise maintenance, it could therefore be saved in order to preserve mental effort (Dietrich \& Audiffren, 2011). Several theoretical propositions have been formulated to indicate that a modulation in attention could influence the maintenance of exercise (Rejeski, 1985; Tucker, 2009). For example, Rejeski suggested that the rating of perceived exertion (RPE), the feeling of effort experienced during a physical task (Borg, 1982), would be reduced if attention were not focused on the physical task. In line with this model, it has been shown that RPE is lower during prolonged exercise when one is listening to music, in comparison with a control condition (Yamashita, Iwai, Akimoto, Sugawara, \& Kono, 2006), suggesting that distraction from the physical task can reduce the feeling of effort. Using a more controlled method in which participants performed the same exercise in the same conditions, Baden and colleagues (Baden, WarwickEvans, \& Lakomy, 2004, Study 1) found that participants' attention was directed more away from the physical task when they expected to run a long rather than a short distance. In addition, RPE was less elevated in the expectation of a long than of a short run. These results suggest that attentional focus might be naturally turned away from the physical task to 
reduce the feeling of effort when prolonged exercise is expected. However, it remains unknown whether such psychological adaptation to prolonged exercise anticipation is accompanied by a modulation of brain activity.

Top-down attention and executive control are supported by a set of brain areas that constitute the central executive network (CEN). Specifically, functional magnetic resonance imaging (fMRI) studies have shown that the engagement of attentional resources activates brain regions mainly located in a fronto-parietal network such as the dorsolateral prefrontal cortex (dIPFC), the inferior frontal cortex (IFC), or the posterior parietal cortex (e.g., Corbetta \& Schulman, 2002; Esterman, Rosenberg, \& Noonan, 2014; Johnson \& Zatorre, 2006; Pardo, Fox, \& Raichle, 1991). Interestingly, brain activity during attentional effort is characterized not only by increased activity in CEN regions, but also by decreased activity in other regions. Regions such as the posterior cingulate cortex, the medial prefrontal cortex (mPFC), and the lateral parietal cortex are down-regulated during attentional effort (e.g., Esterman et al., 2014; Raichle et al., 2001), and these regions are among the main constituents of the default mode network $(\mathrm{DMN})$. Functional connectivity analyses indicate that the CEN and the DMN work mostly in an anticorrelated fashion (Fox, Zhang, Snyder, \& Raichle, 2009; Greicius, Krasnow, Reiss, \& Menon, 2003). The DMN is typically activated when individuals are at rest and not engaged in a specific task (Raichle et al., 2001). Further fMRI studies have shown that even when individuals are not actively engaged in a task, their minds are not passive, since regions of the DMN are activated during self-related introspection or while processing emotional stimuli and thinking about others' mental states (e.g., Blakemore, 2008; Gusnard, Akbudak, Shulman, \& Raichle, 2001). In other words, the DMN is thought to be associated with mind-wandering states (Christoff, Gordon, Smallwood, Smith, \& Schooler, 2009). It should be noted that mind wandering is known to occur not only during rest, but also during prolonged or repetitive tasks in which lapses of attention are frequent (Kane et al., 2007).

Theoretical predictions have not yet indicated specific activation of the CEN or the DMN during exercise. The reticular-activating hypofrontality (RAH; Dietrich \& Audiffren, 2011) model, one of the most commonly used models to account for the effect of exercise on brain activity, suggests that exercise progressively leads to a general downregulation of the prefrontal cortex (PFC). On the basis of Ide and Secher's (2000) observation that global cerebral blood flow does not increase during prolonged exercise, in spite of the ongoing activity of motor brain regions (e.g., motor cortex, supplementary motor area, the cerebellum), Dietrich and Audiffren proposed that nonmotor areas, and particularly the PFC, should be disengaged during exercise. Attentional capacity and executive performance would therefore be diminished during exercise, and even more so in the case of prolonged exercise, which would impose greater constraints on the central nervous system. The empirical evidence for hypofrontality during exercise has been mixed, however. Concerning behavioral performance of attentional or executive tasks, some studies have reported decreased performance during exercise (e.g., Del Giorno, Hall, O’Leary, Bixby, \& Miller, 2010; Wang, Chu, Chu, Chan, \& Chang, 2013), but others have reported improved performance during prolonged exercise at moderate intensity (Pesce, Capranica, Tessitore, \& Figura, 2003) or at high intensity (e.g., Davranche, Brisswalter, \& Radel, 2015; Schmit et al., 2015). In recent years, studies have increasingly recorded brain activity during exercise and can now provide a more direct assessment of the hypofrontality hypothesis. Most of these studies have been conducted using near-infrared spectroscopy (NIRS), because NIRS is relatively tolerant of motion (Perrey, 2008). As a hemodynamic measure, NIRS, like fMRI, monitors functional brain activity indirectly. Specifically, changes in the concentrations of oxyhemoglobin $\left(\mathrm{O}_{2} \mathrm{Hb}\right)$ measured by NIRS rely on the detection of neurovascular coupling to infer changes in neuronal activity (Perrey, 2008; Quaresima, Bisconti, \& Ferrari, 2012). Although a meta-analysis of NIRS studies conducted during incremental exercise indicated an elevation of $\mathrm{O}_{2} \mathrm{Hb}$ in the PFC that increased over time or with intensity (Rooks et al., 2010), a marked $\mathrm{O}_{2} \mathrm{Hb}$ decrease has often been reported before exhaustion at very high intensities (e.g., Bhambhani, Malik, \& Mookerjee, 2007; Rupp \& Perrey, 2008; see also Ekkekakis, 2009, for a review of the NIRS response during exercise). This typical pattern occurring in the last part of a maximal exercise test was shown to be located in the right dlPFC (Tempest, Eston, \& Parfitt, 2014). However, it should be noted that these studies were based on incremental exercise, which makes it impossible to dissociate the time effects from the intensity effects, since time and intensity increase simultaneously in such protocols. To better determine the neurocognitive adaptations that occur over time to deal with the maintenance of physical exercise, NIRS investigations should instead rely on tasks with a constant workload (e.g., Giles et al., 2014).

The aim of the present study was to investigate the neurocognitive changes that arise when coping with the expectation of prolonged exercise. Given that mental effort is a limited resource (Marcora, Staiano, \& Manning, 2009; Muraven \& Baumeister, 2000), we assumed that individuals might save mental effort when they expected to exercise for a long duration. To test this hypothesis, we used the deception approach proposed by Baden et al. (2004; see also Eston, Stansfield, Westoby, \& Parfitt, 2012), in which participants performed the same exercise twice, but with different expectations of the duration of the exercise trial (10 vs. $60 \mathrm{~min})$. Whereas the RAH model predicts a disengagement of the PFC during prolonged exercise without making a distinction between PFC regions, we predicted that only the regions 
associated with the CEN (i.e., lateral PFC) would be downregulated during the prolonged exercise. NIRS monitoring was chosen because of its compatibility with exercise. It should also be recalled that NIRS can successfully dissociate brain activity related to attentional focus from brain activity related to task-unrelated thoughts (Durantin, Dehais, \& Delorme, 2015; Harrivel, Weissman, Noll, \& Peltier, 2013; Stevenson, Russell, \& Helton, 2011). Given the limitation of NIRS in terms of both depth sensitivity ( $\sim 20 \mathrm{~mm}$; Haeussinger et al., 2011) and spatial resolution ( $\sim 10 \mathrm{~mm}$; Quaresima et al., 2012), we selected two regions of interest that are accessible by NIRS (i.e., are located on the surface of a cortical gyrus) and that are sufficiently separated from each other to prevent contamination. Specifically, one NIRS channel was placed over the right dIPFC (rdlPFC), since it is a representative region of the CEN (e.g., Johnson \& Zatorre, 2006; Pardo et al., 1991), and the other over the right dorsomedial PFC (rmPFC), since this region has been associated with the DMN (Eickhoff, Laird, Fox, Bzdok, \& Hensel, 2016). Two right channels were chosen because the attentional network is thought to be more localized in the right hemisphere (Corbetta \& Schulman, 2002). We expected that activity in the rdIPFC would be lower in the 60-min than in the 10-min condition, and that activity in the rmPFC would be higher in the 60-min than in the 10-min condition. We predicted that participants' attentional focus would be more directed away from the physical task in the 60-min condition, leading to a reduced RPE as compared with the 10-min condition.

\section{Method}

\section{Participants}

To determine the sample size, we performed a sample size calculation that adjusted for the correlated nature of the repeated measurements (Liu \& Liang, 1997). The power analysis was performed with PASS (PASS 14, NCSS, Kaysville, USA) using a simulation approach. The significance level was fixed at $p=.05$ and the power level was fixed at .80 . We inferred effect size from the study by Baden et al. (2004), in which the deception manipulation led to a $19.6 \%$ change in attentional focus. Using these parameters, and after 1,000 simulations, the power analysis indicated that at least 20 participants were needed for this study. Twenty-two students (15 males and seven females, $M_{\text {age }}=21.27 \pm 2.07$ years) at the University of Nice Sophia-Antipolis were recruited in exchange for course credits. To be eligible for the study, the participants had to report at least one session of moderate long-duration exercise per week (e.g., biking or running for more than $1 \mathrm{~h}$ ). Table 1 presents the participants' anthropometric and physiological characteristics. All provided written informed consent,
Table 1 Anthropometric and physiological characteristics of the participants

\begin{tabular}{llll}
\hline & All & Women & Men \\
\hline Sample size & 22 & 7 & 15 \\
Age [years] & $21.3 \pm 2.1$ & $23.0 \pm 1.9$ & $20.5 \pm 1.6$ \\
Height [cm] & $174.7 \pm 6.4$ & $168.6 \pm 6.2$ & $177.6 \pm 4.3$ \\
Body mass [kg] & $67.0 \pm 10.6$ & $53.1 \pm 2.9$ & $73.5 \pm 4.9$ \\
Peak HR [bpm] & $186.5 \pm 10.9$ & $191.0 \pm 9.5$ & $184.3 \pm 11.2$ \\
PAP [watts] & $286.1 \pm 79.3$ & $200.0 \pm 68.8$ & $310.7 \pm 64.7$ \\
\hline
\end{tabular}

$S D=$ standard deviation $; \mathrm{HR}=$ heart rate $\mathrm{PAP}=$ peak aerobic power

and the study was conducted according to the Declaration of Helsinki.

\section{Experimental procedure}

The participants came to the lab for three sessions. Before each session, they were instructed to abstain from any vigorous exercise for $24 \mathrm{~h}$ pre-session, to sleep at least $7 \mathrm{~h}$ the night before, and to avoid changes in caffeine ingestion in the morning before the experiment.

In the first session, they signed the informed consent form and were asked to perform an incremental test to exhaustion on an electromagnetically braked cycle ergometer (Brain-bike NeuroActive, Motion Fitness, Rolling Meadows, IL, USA), to identify their peak aerobic power (PAP) and peak heart rate (HR). The power output was increased by $20 \mathrm{~W}$ every minute after a 4-min warm-up at light intensity (women, $70 \mathrm{~W}$; men, $80 \mathrm{~W}$ ). Voluntary exhaustion was defined as the point at which participants voluntarily stopped or they could no longer maintain a pedaling frequency above 50 rotations per minute for more than $10 \mathrm{~s}$, despite strong verbal encouragement. Heart rate was recorded continuously during the incremental test by a Polar system (Polar RS800CX, Polar Electror Oy, Kempele, Finland). After the test, the participants were also familiarized with the two psycho/physical scales to be used in the subsequent experimental trials (attentional focus and RPE).

The two experimental sessions were held at the same time of day, with a mean interval of $3.2 \pm 1.4$ days. The participants were informed that they would perform exercise at an intensity corresponding to $60 \%$ PAP on the cycle ergometer and that this would last for either 10 or $60 \mathrm{~min}$, according to the condition. The order of the two trials was counterbalanced. Before starting the exercise, they were fit with the NIRS cap and the Polar chest belt. A 2-min baseline period followed in which they were seated without movement on the ergometer. They then performed a 1-min warm-up at low intensity $(70 \mathrm{~W}$ for females and $80 \mathrm{~W}$ for males), which was followed by a 4min period in which the intensity progressively increased to the target intensity. Although the ergometer adjusted the power output to the pedaling frequency, the participants were 
asked to stay at a fixed pedaling frequency all along the trial, which was similar for the two experimental sessions. Three sound probes were given at 200, 400, and $600 \mathrm{~s}$ after the trial onset. Each probe was immediately followed by two questions displayed on a screen located in front of the participants to assess their attentional focus and RPE. In both experimental sessions, the trial was stopped after $10 \mathrm{~min}$. The experimenter justified the early stop in the 60 -min condition by saying that a sufficient amount of data had been collected, so that the exercise could be stopped sooner than planned. At the end of the study, all participants were thanked and carefully debriefed about the aims of the study. The experimenter also explained the necessary use of deception in the method and asked the participants not to communicate this information to other possible participants, to avoid a contamination effect.

\section{Measures}

NIRS Cerebral hemodynamics were measured in two subregions of the PFC using a continuous-wave multi-channel NIRS (Oxymon Mk II, Artinis Medical Systems, Zetten, the Netherlands). Optodes were placed into thermoplastic shells and mounted on a head cap (Easycap, Herrsching, Germany) to facilitate the positioning of the optodes on each participant's head. To maintain sufficient pressure on the optodes at the skin surface, elastic bands were inserted on the probe holders to push the probes into the holders. NIRS sensitivity to gray matter depends to the source-detector distance and head region (Quaresima et al., 2012; Strangman, Zhang, \& Li, 2014). Since penetration of light is elevated in the frontal region in comparison with other regions due to the absence of hair, the distance between the source and detector probe was fixed at $4.0 \mathrm{~cm}$. This distance allows for deeper recording, which should ensure that a significant part of the NIRS signal comes from a cortical source. Strangman et al. determined that a 4.0$\mathrm{cm}$ source-detector distance on the frontal cortex yields high NIRS sensitivity to gray matter in the frontal region ( 15\% of the NIRS signal). The area measured between the emitter and detector probes was defined as a channel. The positioning of the probes was arranged so that the two NIRS channels were centered on the AF2 $h$ and F6h sites of the extended 10-5 EEG system (Jurcak, Tsuzuki, \& Dan, 2007). The cortical structure below the AF2h site corresponds to the anterior part of the right dorsomedial PFC (BA10). The cortical structure below the F6h site corresponds to the rdlPFC on the middle frontal gyrus (BA46). The projection of the positions of the two channels on the cortical surface (based on their stereotaxic coordinates) is illustrated in Fig. 1. The concentrations (in micromoles) of $\mathrm{O}_{2} \mathrm{Hb}$ and deoxyhemoglobin were provided using two wavelengths in the near-infrared range (764 and $858 \mathrm{~nm}$ ) and an age-specific differential path length factor obtained using the modified Beer-Lambert equation (Obrig \& Villringer, 2003). The data were sampled at $10 \mathrm{~Hz}$ and

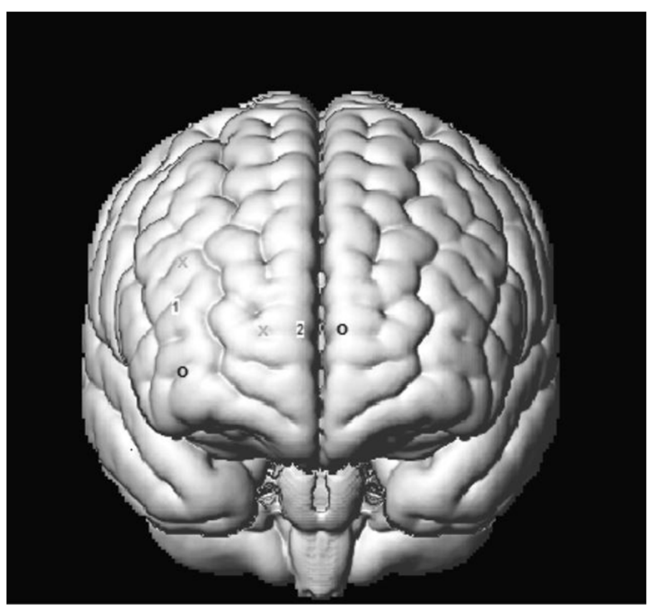

Fig. 1 Locations of near-infrared spectroscopy (NIRS) channels, through projection on the cortical surface using the NIRS-SPM software (Ye, Tak, Jang, Jung, \& Jang, 2009). The probes were arranged to measure hemodynamic activity in two regions of interest: the right dorsolateral prefrontal cortex (Channel 1) and the right medial frontal cortex (Channel 2).

acquired with Oxysoft (version 3.0.43; Artinis Medical Systems, Zetten, The Netherlands). We computed an average $\mathrm{O}_{2} \mathrm{Hb}$ value for each of the three time periods during exercise $(0-200,200-400$, and 400-600 s) at the target intensity $(60 \%$ PAP). The moment during which the participants answered the two questions was excluded from these time windows.

Attentional focus The attentional focus was assessed by adapting the procedure of Christoff et al. (2009) and Baden et al. (2004). After a sound probe, the following question was immediately displayed on a screen in front of each participant: "Where was your attention focused before the probe?" With a mouse click placed within reach of their right hand, the participants had to provide their response on a visual analog scale (VAS) displayed on the screen. The scale ranged from completely on task to completely off task. During the familiarization period, the participants were instructed that a minimal value on this scale indicated that they had spent all the period preceding the probe thinking about the exercise task they were performing, and that a maximal value indicated that they had spent all their time thinking about something else. Several examples were provided to ensure correct understanding of the scale. The position of the cursor was recorded in pixels and was then converted to a $0-100$ scale to provide a meaningful score.

RPE A second question then followed to assess the participants' perceived exertion. To optimize the sensitivity of the measure and to avoid any memory of previous responses, participants also had to provide their response with a mouse click on a VAS, a valid measure of perceived exertion (Grant et al., 1999). The VAS had the same labels as the original 6-20 Borg scale (Borg, 1970). The cursor positions (in pixels) were 
then converted to the 6-20 RPE units in order to provide a meaningful interpretation of the recorded values.

\section{Data analysis}

We focused on $\mathrm{O}_{2} \mathrm{Hb}$ as the variable of interest to determine changes in PFC activity (Yamanaka, Yamagata, Tomioka, Kawasaki, \& Mimura, 2010). Changes in $\mathrm{O}_{2} \mathrm{Hb}$ signals have been recognized to better reflect cortical activation than $\mathrm{dHb}$ due its superior contrast-to-noise ratio (Strangman et al., 2002). In addition, previous independent studies provide evidence for fNIRS $\mathrm{O}_{2} \mathrm{Hb}$ being an appropriate and better substitute for fMRI BOLD for studying cortical activity related to cognitive and motor tasks (Muthalib et al., 2013; Steinbrink et al., 2006; Strangman et al., 2002). To process the NIRS signals, we first checked for the quality of the signals by calculating the signal-to-noise ratio (SNR) using an adaptation of the formula used by Cui, Bray, and Reiss (2010):

$$
\begin{aligned}
\mathrm{SNR}= & \text { mean }(\text { exercise })-\operatorname{mean}(\text { baseline }) / \\
& \times \sqrt{\lfloor\text { variance }(\text { exercise }+ \text { baseline })\rfloor} .
\end{aligned}
$$

The NIRS signals were then filtered using a fourth-order digital low-pass Butterworth filter with a cutoff frequency of $0.1 \mathrm{~Hz}$, to remove the HR and respiratory components (Huppert, Diamond, Franceschini, \& Boas, 2009). The data were subtracted from baseline to express the magnitude of the changes from baseline (representing $0 \mu \mathrm{M}$ ). To reduce intersubject variability, the data were also normalized by dividing all $\mathrm{O}_{2} \mathrm{Hb}$ values by their range (the difference between the maximal and the minimal values of an individual). The distributions of all dependent variables were verified by visual inspection of the frequency histograms, box plots, and quantile-quantile plots. To examine the hypothesis related to changes in brain activity, a linear mixed model (LMM) approach was used controlling for the nonindependence of the data by including a random intercept effect structured by participants. This approach brings a much higher level of statistical power level than does traditional repeated measures analysis of variance (Ma, Mazumdar, \& Memtsoudis, 2013). In addition, another advantage is the possibility to control for all specific characteristics related to the conditions and to the individuals (Hoffman \& Rovine, 2007). In our models, we entered the order of the conditions, peak HR, and the sex of the participants as fixed effects. In the LMM on $\mathrm{O}_{2} \mathrm{Hb}$, a Channel factor (brain region: rdlPFC and rmPFC), a Time factor (average concentrations at 0-200, 200-400, and 400$600 \mathrm{~s}$ ), a factor representing the Experimental Condition (expected duration: 10 and $60 \mathrm{~min}$ ), and all possible interactions between these three factors were entered as fixed effects. To examine the hypotheses about the subjective measures, two separate LMMs were conducted on attentional focus and RPE, with a factor representing the Time Period (0-200, 200-400, and 400-600 s) and a factor representing the experimental condition (Expected Duration: 10 and $60 \mathrm{~min}$ ) as fixed effects, in addition to gender and the order of conditions. Effects were considered significant when the $p$ value was below .05, and marginally significant when below.10. If effects were at least marginally significant, we interpreted interaction effects using post-hoc comparisons of the estimated means generated by the LMM. A correlation matrix between self-reported and hemodynamics measures was also performed for exploratory purposes. All statistics were performed using SPSS (version 23, IBM Corp., Armonk, NY).

\section{Results}

For the changes in hemodynamic concentration, the analysis of the signal quality indicated acceptable values on both channels for almost all participants (mean SNR $=3.284$ ). Only one participant was excluded from further analysis due to the very low quality of the NIRS signal on both channels $($ SNR $<1$ ). Figure 2 presents the trace of one of the participants, to illustrate the pattern changes in the NIRS signal for each phase of the protocol.

Figure 3 presents the average $\mathrm{O}_{2} \mathrm{Hb}$ concentrations in the three periods of the exercise trial in each experimental condition for the rdIPFC and rmPFC. The results of the LMM on $\mathrm{O}_{2} \mathrm{Hb}$ revealed a significant main effect of time, $F(2,219)=$ $12.142, p=.001$, indicating a lower general $\mathrm{O}_{2} \mathrm{Hb}$ value in the first period $(0.050 \mu \mathrm{M})$ than in the second $(0.227 \mu \mathrm{M})$ and third $(0.276 \mu \mathrm{M})$ periods of time $(p \mathrm{~s}<.001)$. A significant two-way interaction between channel and condition was also found, $F(1,219)=10.235, p=.002 . \mathrm{O}_{2} \mathrm{Hb}$ concentrations in the rdIPFC were less elevated in the 60-min than in the 10-min condition $(0.142$ vs. $0.297 \mu \mathrm{M}, p=.001)$, whereas $\mathrm{O}_{2} \mathrm{Hb}$ concentrations in the rmPFC were higher in the 60-min than in the 10 -min condition $(0.197$ vs. $0.100 \mu \mathrm{M}, p=.036)$. No other effects were found.

The LMM conducted on attentional focus only revealed a significant effect of the condition, $F(1,105)=7.606, p=.007$. As is shown in Fig. 4, participants' attention was less focused on the exercise trial in the 60-min than in the 10-min condition (46.26 vs. 38.43 ).

Concerning RPE (Fig. 5), the LMM did not reveal any significant or marginally significant effects.

Concerning the correlations between the hemodynamics and the subjective measures (Table 2), a significant negative correlation was found between the $\mathrm{O}_{2} \mathrm{Hb}$ concentrations on the channel located in the rdIPFC and the measures of attentional focus. Since a low value on the attentional focus scale represents an attentional focus on the exercise task, this suggests that the $\mathrm{O}_{2} \mathrm{Hb}$ concentration in the rdlPFC was higher when the participants reported being focused on the exercise 


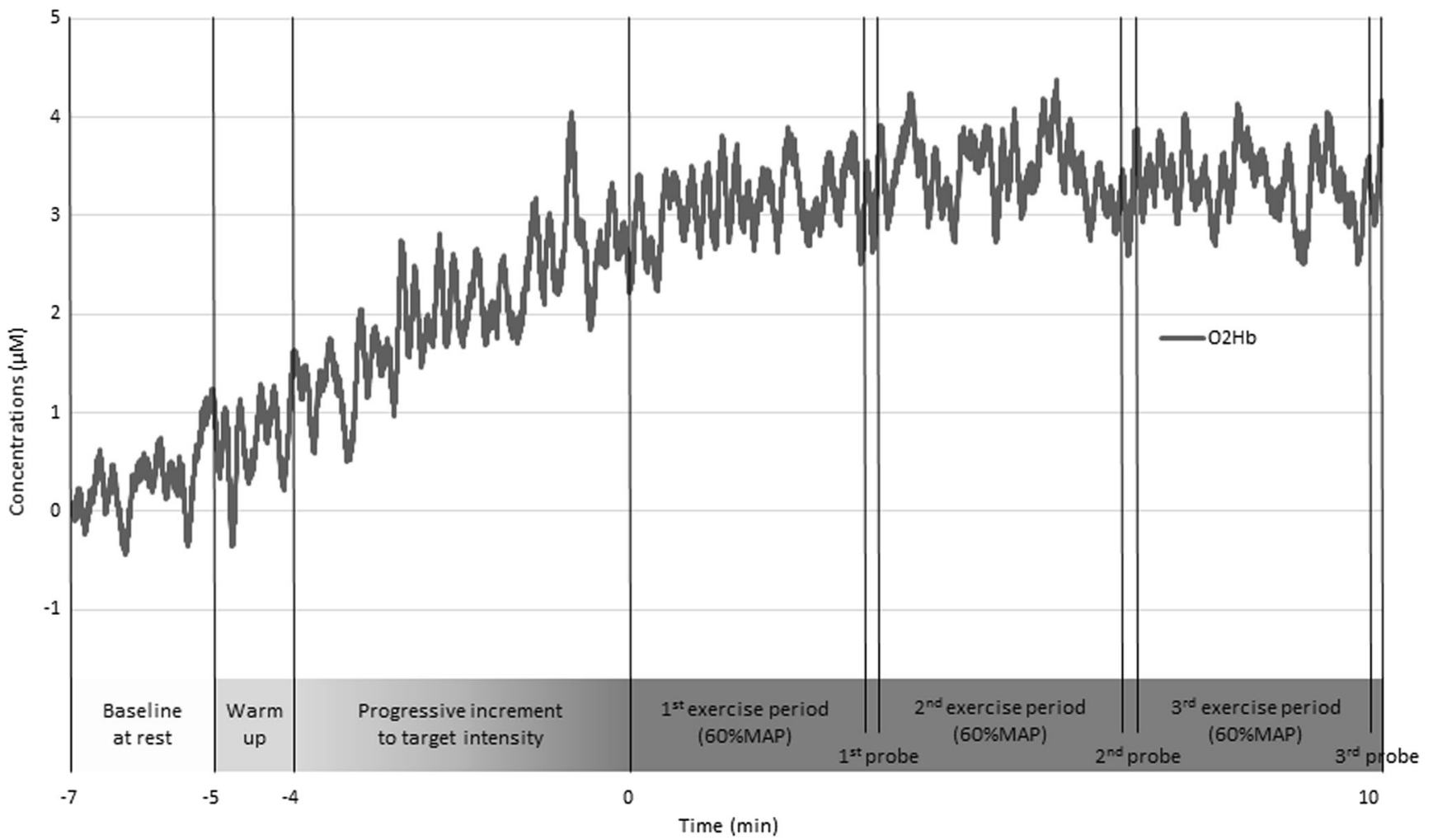

Fig. 2 Illustration of changes in $\mathrm{O}_{2} \mathrm{Hb}$ concentrations over an entire experimental session. The raw data are taken from the recording of the channel located in the right dorsolateral prefrontal cortex during the first

experimental session (an announced duration of $10 \mathrm{~min}$ ) for one representative participant in the experiment.

task. No correlation was found between the $\mathrm{O}_{2} \mathrm{Hb}$ concentrations measured at the channel located on the right $\mathrm{mPFC}$ and the two subjective measures. The two subjective measures were significantly associated: Higher RPE scores were associated with a focus on the exercise task.

\section{Discussion}

The objective of this study was to investigate the neurocognitive changes that arise when coping with presumably prolonged exercise. The results confirmed the findings

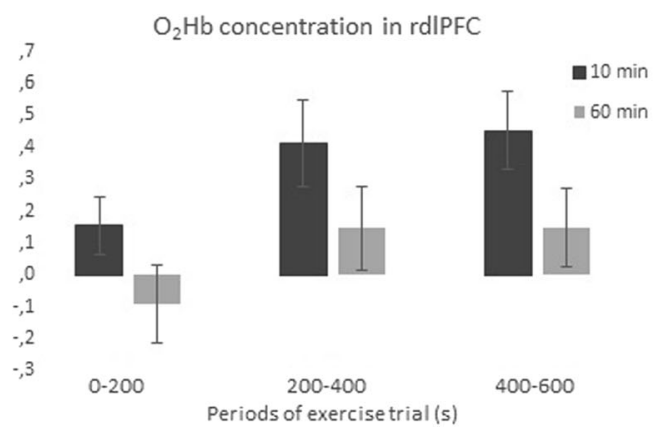

Fig. 3 Oxyhemoglobin $\left(\mathrm{O}_{2} \mathrm{Hb}\right)$ concentrations in the three periods of the exercise trial as a function of the experimental condition (expected duration of either $10 \mathrm{~min}$ or $60 \mathrm{~min}$ of exercise). The left panel depicts the concentrations in the right dorsolateral prefrontal cortex (rdlPFC), and reported in deception studies that have manipulated the announced duration of the exercise trial, by indicating the existence of strategic adaptations to cope with the expectation of a longer exercise duration (Baden et al., 2004; Baden et al., 2005; Eston et al., 2012; Gabbett, Walker, \& Walker, 2015). For example, expecting to exercise for a longer duration has led to a decrease in attentional focalization on the exercise (Baden et al., 2004, Study 1), associated with lower RPE (Baden et al., 2004; Eston et al., 2012) and a lower affect score. More generally, these results suggest that such expectations of physical exercise can have an important influence on the subjective experience of the physical task (see also

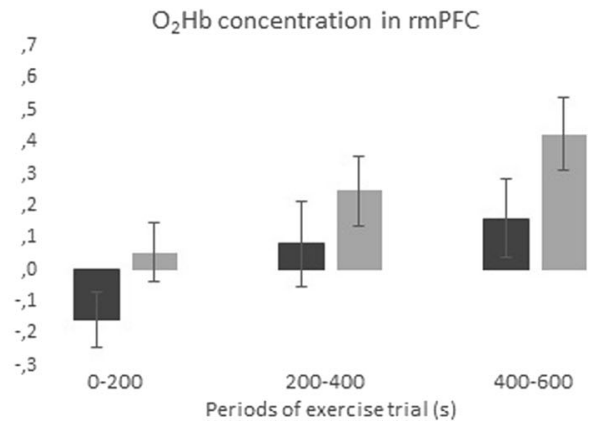

the right panel represents concentrations in the right medial prefrontal cortex (rmPFC). Values are means, with error bars representing the standard errors of the means. $N=21$ participants 


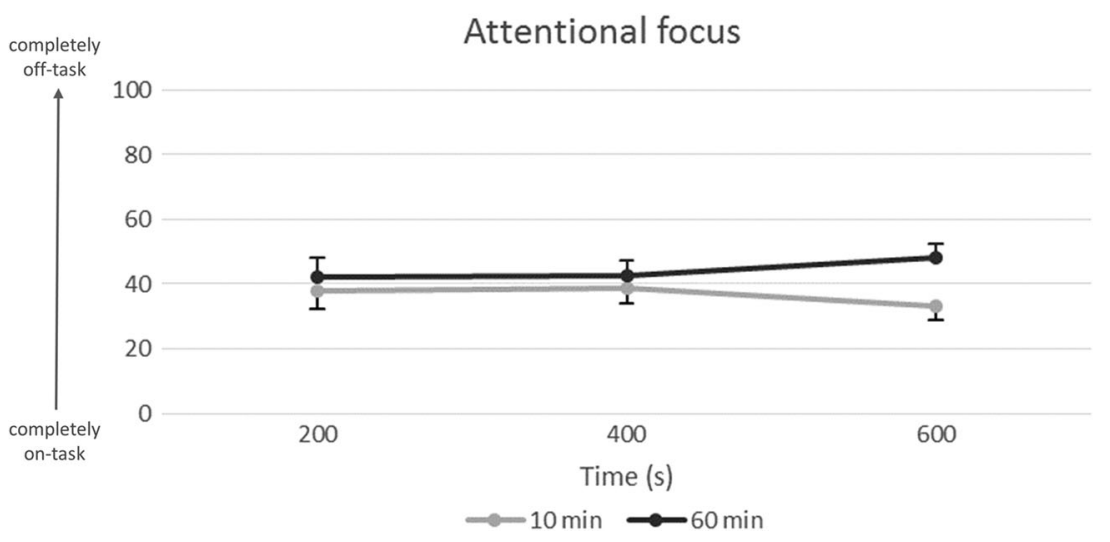

Fig. 4 Attentional focus scores across time as a function of the experimental condition (expected duration of either $10 \mathrm{~min}$ or $60 \mathrm{~min}$ of exercise). A low score indicates a focus on the exercise trial, and a

Buman, Brewer, \& Cornelius, 2009). But unlike these previous results, our study has been the first to show an influence of the expected duration on brain activity, assessed via NIRS during the physical task.

To discuss the cerebral hemodynamic results, it is first important to note that we observed a general increase in cerebral oxygenation, as reflected by $\mathrm{O}_{2} \mathrm{Hb}$ changes in both channels during exercise. This is in accordance with the general results of the meta-analysis by Rooks et al. (2010), that whole-body exercise increases cerebral oxygenation. Besides this general increase in time, we observed a different response pattern between the 10-min and 60-min conditions in $\mathrm{O}_{2} \mathrm{Hb}$. Because $\mathrm{O}_{2} \mathrm{Hb}$ is presumed to increase as a metabolic adjustment in response to neural firing (Obrig \& Villringer, 2003), this pattern suggests that the manipulation (i.e., solely the announced information about the duration of the exercise trial) led to real modifications in brain activity. Indeed, the manipulation induced differences in the rdIPFC and the rmPFC, indicating regional variations within the PFC. Specifically, $\mathrm{O}_{2} \mathrm{Hb}$ concentrations in the rdlPFC was less elevated in the

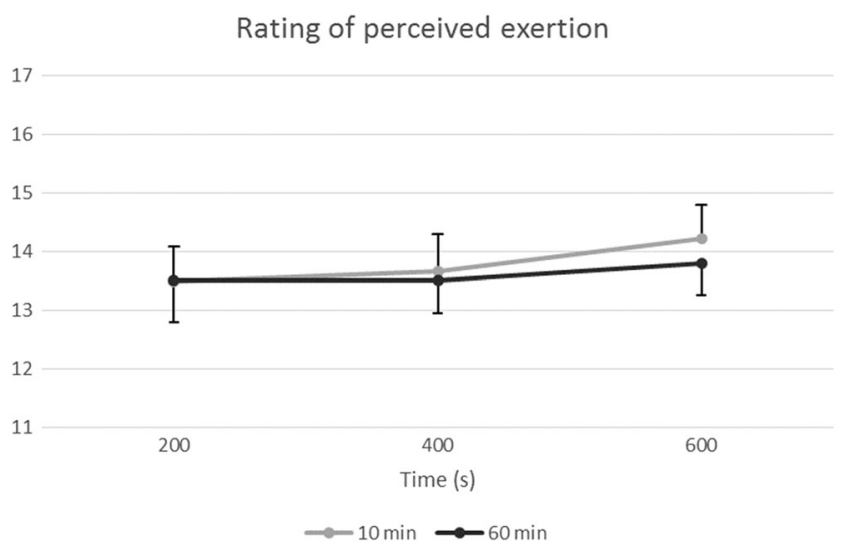

Fig. 5 Rating-of-perceived exertion scores across time as a function of the experimental condition (expected duration of either $10 \mathrm{~min}$ or $60 \mathrm{~min}$ of exercise). Values are means, with error bars representing the standard errors of the means. $N=22$ participants high score indicates a high amount of thinking unrelated to the exercise trial. Values are means, with error bars representing the standard errors of the means. $N=22$ participants

60-min than in the 10-min condition, but in the rmPFC, $\mathrm{O}_{2} \mathrm{Hb}$ concentrations were higher in the 60-min than in the 10-min condition (see Fig. 3). Since rdlPFC is a representative region of the CEN and rmPFC is a representative region of the DMN, this result is consistent with previous fMRI studies that have indicated anticorrelated functioning of these two neural networks (e.g., Fox et al., 2009). Using a quite different method from the aforementioned study, which was based on restingstate functional connectivity, our study also suggests the same pattern. For example, the activity of the CEN was reduced, but DMN activity was greater, during exercise that was expected to last longer.

Interestingly, the findings on the subjective reports of attentional focus (Fig. 4) indicated a reduced focus on the physical task in anticipation of a longer physical effort. In other words, expecting a longer exercise duration led to lower attentional focus on the task (a function supported by the CEN). Since DMN activity has been associated with mind wandering (Christoff et al., 2009), the latter finding certainly occurred more frequently during the exercise condition that was presented as challenging, prolonged exercise. However, the absence of an effect of the manipulation on RPEs did not support the hypothesis that this strategic adaptation occurs to reduce the feeling of exertion while performing the task. This hypothesis was built on the basis of the observation that attentional effort, unlike mind wandering, is difficult to maintain for an extended time (Inzlicht \& Schmeichel, 2012; Muraven \& Baumeister, 2000) and contributes to the conscious feeling of exertion that might be associated with the ability to maintain exercise (e.g., Halson et al., 2002; Marcora, 2010). Therefore, one possible explanation for the lack of an effect on RPE is that the attentional-defocusing strategy was not sufficient to reduce the conscious feeling of exertion in the context of our study. This might have occurred because the exercise trial $(10 \mathrm{~min}$ ) was too short. Even if we did not find a three-way interaction with time, numerical values suggest that the effect built up over time and might have been stronger after 
Table 2 Correlation matrix between average oxyhemoglobin $\left(\mathrm{O}_{2} \mathrm{Hb}\right)$ in the right dorsolateral prefrontal cortex (rdlPFC) and right medial prefrontal cortex (rmPFC), attentional focus, and ratings of perceived exertion (RPE)

\begin{tabular}{|c|c|c|c|c|}
\hline & $\mathrm{O}_{2} \mathrm{Hb}$ rdlPFC & $\mathrm{O}_{2} \mathrm{Hb} \mathrm{rmPFC}$ & Attentional Focus & RPE \\
\hline $\mathrm{O}_{2} \mathrm{Hb}$ rdlPFC & 1 & & & \\
\hline $\mathrm{O}_{2} \mathrm{Hb} \mathrm{rmPFC}$ & .118 & 1 & & \\
\hline Attentional focus & $-.262^{*}$ & -.067 & 1 & \\
\hline RPE & .047 & -.099 & $-.307^{* *}$ & 1 \\
\hline
\end{tabular}

$N=2$ conditions $\times 3$ periods $\times 22$ participants $=132$ measures for attentional focus and RPE, and $N=2$ conditions $\times 3$ periods $\times 21$ participants $=126$ measures for $\mathrm{O}_{2} \mathrm{Hb}$ in rdIPFC and rmPFC

${ }^{*} p=.05,{ }^{* * *} p=.001$

a longer time. Further studies will be needed to better understand the role of attentional focus on the conscious feeling of exertion. Since participants did not provide high values of RPE during the physical exercise, it is also possible that there was no need to reduce their feelings of exertion. In addition to the duration, the physical exercise itself was not intense enough to induce differences in RPE.

We think that the present study has some important implications for understanding the neurocognitive changes that arise during prolonged exercise. As we noted in the introduction, an influential theoretical prediction concerns the progressive down-regulation of the PFC during exercise (Dietrich \& Audiffren, 2011). Our study was built on this framework, and our results seem to refine this theoretical proposition in two directions. First, we assume that hypofrontality mostly results not from an objective limitation of metabolic resources, but instead from a strategic conservation of mental effort resources. As was indicated by Nybo and Secher (2004), it seems that only extreme conditions restrict cerebral metabolism, and thereby cause cognitive perturbation. If this is so, in all other exercise conditions (the vast majority of the time), the regional cerebral blood flow required by the main motor regions would be adequately supplied by the global perfusion without entering into conflict with other regions such as the PFC. However, in addition to this metabolic restriction, it is possible that some prefrontal-dependent functions are downregulated to save the mental effort and executive resources that are needed to maintain the physical task. From that perspective, people should usually be able to perform well at cognitive tasks that rely on prefrontal areas if they are asked to do so (because all metabolic resources are available). But attentional effort would not be engaged if it were not strictly required, according to an effort conservation principle (Silvestrini \& Gendolla, 2013). In fact, the limited capacity of mental effort may not reflect a depletable resource, but rather some preference of the system to avoid cognitive demand (e.g., Inzlicht \& Schmeichel, 2012; Kurzban, Duckworth, Kable, \& Myers, 2013). It is interesting to note that this effort conservation principle is thought of as an automatic rather than an intentional strategy (Silvestrini \&
Gendolla, 2013). In sum, we hypothesized that some prefrontal areas can be down-regulated very early if prolonged exercise is expected. We presume that such down-regulation occurred for the purpose of saving mental effort, even if no data were collected in the frame of this study to test this resource conservation hypothesis. Below, we make suggestions about the specific parts of the PFC that might be down-regulated when prolonged exercise is anticipated.

The second direction in which the different patterns found within the PFC can refine the hypofrontality theory concerns the differentiation of prefrontal structures. Although Dietrich and Audiffren (2011) made predictions about the PFC as a whole, distinctions can be made within the PFC, because its different subregions serve distinct functions and present different activation patterns. For example, in the frame of this study, we distinguished between the right lateral regions involved in the CEN and the right medial regions involved in the DMN, and found distinct forms of activation. Although it is unknown if our results may be extended to the left hemisphere, this distinction is surely important to take into consideration for modeling the effects of exercise on brain activity. Specifically, we believe that if any part of the PFC is downregulated during exercise, this is certainly the regions involved in the CEN (i.e., IFC, dlPFC), since they support functions that are perceived as costly and cannot be maintained for a long time (e.g., Faber, Maurits, \& Lorist, 2012; Muraven \& Baumeister, 2000). Nevertheless, more multichannel NIRS studies with constant workload protocols will be needed to support this idea. Although in the present study we used only two channels, targeted on representative PFC regions of the CEN and the DMN, future studies might also use more NIRS channels to obtain larger coverage of other cortical regions belonging to these two networks.

To conclude, this study provides novel findings showing that neurocognitive changes can occur during the performance of the same physical task in response to differential information about the task duration. We found that both the activity in a brain region associated with attention and mental effort (rdlPFC) and reports of attentional focus on the exercise task decreased in the condition in which a longer exercise duration 
was expected, and we speculate that this finding reveals a process of mental resource conservation. Since people have to use mental resources during prolonged exercise to inhibit the pressure to stop that derives from fatigue-related signals, it makes sense to save some of their limited frontal-executive resources.

Author note The authors thank Laura Gray for her help with data collection, and Gavin Tempest for proofreading. This study was carried out with the support of the Agence Nationale de la Recherche (Grant No. ANR-13-JSH2-0007).

\section{References}

Audiffren, M., \& André, N. (2015). The strength model of self-control revisited: Linking acute and chronic effects of exercise on executive functions. (2015). Journal of Sport and Health Science, 4, 30-46.

Baden, D. A., McLean, T. L., Tucker, R., Noakes, T. D., \& St Clair Gibson, A. (2005). Effect of anticipation during unknown or unexpected exercise duration on rating of perceived exertion, affect, and physiological function. British Journal of Sports Medicine, 39, 742746.

Baden, D. A., Warwick-Evans, L. A., \& Lakomy, J. (2004). Am I nearly there? The effect of anticipated running distance on perceived exertion and attentional focus. Journal of Sport and Exercise Psychology, 26, 1-17.

Bhambhani, Y., Malik, R., \& Mookerjee, S. (2007). Cerebral oxygenation declines at exercise intensities above the respiratory compensation threshold. Respiratory Physiology \& Neurobiology, 156, 196-202. doi:10.1016/j.resp.2006.08.009

Blakemore, S.-J. (2008). The social brain in adolescence. Nature Reviews Neuroscience, 9, 267-277. doi:10.1038/nrn2353

Borg, G. (1970). Perceived exertion as an indicator of somatic stress. Scandinavian Journal of Rehabilitation Medicine, 2, 92-98.

Borg, G. (1982). Psychophysical bases of perceived exertion. Medicine and Science in Sports and Exercise, 14, 377-381.

Buman, M. P., Brewer, B. W., \& Cornelius, A. E. (2009). A discrete-time hazard model of hitting the wall in recreational marathon runners. Psychology of Sport and Exercise, 10, 662-666. doi:10.1016/j. psychsport.2009.04.004

Christoff, K., Gordon, A. M., Smallwood, J., Smith, R., \& Schooler, J. W. (2009). Experience sampling during fMRI reveals default network and executive system contributions to mind wandering. Proceedings of the National Academy of Sciences, 106, 8719-8724. doi:10.1073 /pnas.0900234106

Corbetta, M., \& Schulman, G. L. (2002). Control of goal-directed and stimulus-driven attention in the brain. Nature Neuroscience Reviews, 3, 201-215.

Cui, X., Bray, S., \& Reiss, A. L. (2010). Functional near infrared spectroscopy (NIRS) signal improvement based on negative correlation between oxygenated and deoxygenated hemoglobin dynamics. NeuroImage, 49, 3039-3046.

Davranche, K., Brisswalter, J., \& Radel, R. (2015). Where are the limits of the effects of exercise intensity on cognitive control? Journal of Sport and Health Science, 4, 56-63.

Del Giorno, J. M., Hall, E. E., O'Leary, K. C., Bixby, W. R., \& Miller, P. C. (2010). Cognitive function during acute exercise: A test of the transient hypofrontality theory. Journal of Sport and Exercise Psychology, 32, 312-323. doi:10.1123/jsep.32.3.312

Dietrich, A., \& Audiffren, M. (2011). The reticular-activating hypofrontality (RAH) model of acute exercise. Neuroscience \& Biobehavioral Reviews, 35, 1305-1325.
Durantin, G., Dehais, F., \& Delorme, A. (2015). Characterization of mind wandering using fNIRS. Frontiers in Systems Neuroscience, 9, 45. doi: $10.3389 /$ fnsys. 2015.00045

Eickhoff, S. B., Laird, A. R., Fox, P. T., Bzdok, D., \& Hensel, L. (2016). Functional segregation of the human dorsomedial prefrontal cortex. Cerebral Cortex, 26, 304-321. doi:10.1093/cercor/bhu250

Ekkekakis, P. (2009). Illuminating the black box: Investigating prefrontal cortical hemodynamics during exercise with near-infrared spectroscopy. Journal of Sport and Exercise Psychology, 31, 505-553.

Esterman, M., Rosenberg, M., \& Noonan, S. (2014). Intrinsic fluctuations in sustained attention and distractor processing. Journal of Neuroscience, 34, 1724-1730. doi:10.1523/JNEUROSCI.265813.2014

Eston, R., Stansfield, R., Westoby, P., \& Parfitt, G. (2012). Effect of deception and expected exercise duration on psychological and physiological variables during treadmill running and cycling. Psychophysiology, 49, 462-469.

Faber, L. G., Maurits, N. M., \& Lorist, M. M. (2012). Mental fatigue affects visual selective attention. PLoS ONE, 7, e48073. doi:10.1371/journal.pone.0048073

Fox, M. D., Zhang, D., Snyder, A. Z., \& Raichle, M. E. (2009). The global signal and observed anticorrelated resting state brain networks. Journal of Neurophysiology, 101, 3270-3283.

Gabbett, T. J., Walker, B., \& Walker, S. (2015). Influence of prior knowledge of exercise duration on pacing strategies during game-based activities. International Journal of Sports Physiology and Performance, 10, 298-304.

Giles, G. E., Brunyé, T. T., Eddy, M. D., Mahoney, C. R., Gagnon, S. A., Taylor, H. A., \& Kanarek, R. B. (2014). Acute exercise increases oxygenated and deoxygenated hemoglobin in the prefrontal cortex. NeuroReport, 25, 1320-1325. doi:10.1097/WNR.0000000000000266

Grant, S., Aitchison, T., Henderson, E., Christie, J., Zare, S., McMurray, J., \& Dargie, H. (1999). A comparison of the reproducibility and the sensitivity to change of visual analogue scales, Borg scales, and Likert scales in normal subjects during submaximal exercise. Chest, 116, 1208-1217.

Greicius, M. D., Krasnow, B., Reiss, A. L., \& Menon, V. (2003). Functional connectivity in the resting brain: A network analysis of the default mode hypothesis. Proceedings of the National Academy of Sciences, 100, 253-258.

Gusnard, D. A., Akbudak, E., Shulman, G. L., \& Raichle, M. E. (2001). Medial prefrontal cortex and self-referential mental activity: Relation to a default mode of brain function. Proceedings of the National Academy of Sciences, 98, 4259-4264.

Haeussinger, F. B., Heinzel, S., Hahn, T., Schecklmannn, M., Ehlis, A., \& Fallgater, A. (2011). Simulation of near-infrared light absorption considering individual head and prefrontal cortex anatomy: Implications for optical neuroimaging. PLoS ONE, 6, e26377. doi:10.1371/journal.pone.0026377

Halson, S. L., Bridge, M. W., Meeusen, R., Busschaert, B., Gleeson, M., Jones, D. A., \& Jeukendrup, A. E. (2002). Time course of performance changes and fatigue markers during intensified training in trained cyclists. Journal of Applied Physiology, 93, 947-956. doi:10.1152/japplphysiol.01164.2001

Harrivel, A. R., Weissman, D. H., Noll, D. C., \& Peltier, S. J. (2013). Monitoring attentional state with fNIRS. Frontiers in Human Neuroscience, 7, 861. doi:10.3389/fnhum.2013.00861

Hoffman, L., \& Rovine, M. J. (2007). Multilevel models for the experimental psychologist: Foundations and illustrative examples. Behavior Research Methods, 39, 101-117. doi:10.3758 /BF03192848

Huppert, T. J., Diamond, S. G., Franceschini, M. A., \& Boas, D. A. (2009). HomER: A review of time-series analysis methods for near-infrared spectroscopy of the brain. Applied Optics, 48, 280 298. 
Ide, K., \& Secher, N. H. (2000). Cerebral blood flow and metabolism during exercise. Progress in Neurobiology, 61, 397-414.

Inzlicht, M., \& Schmeichel, B. J. (2012). What is ego depletion? Toward a mechanistic revision of the resource model of self-control. Perspectives on Psychological Science, 7, 450-463. doi:10.1177 /1745691612454134

Johnson, J. A., \& Zatorre, R. J. (2006). Neural substrates for dividing and focusing attention between simultaneous auditory and visual events. NeuroImage, 31, 1673-1681.

Jurcak, V., Tsuzuki, D., \& Dan, I. (2007). 10/20, 10/10, and 10/5 systems revisited: Their validity as relative head-surface-based positioning systems. NeuroImage, 34, 1600-1611. doi:10.1016/j. neuroimage.2006.09.024

Kahneman, D. (1973). Attention and effort. Engelwood Cliffs, NJ: Prentice Hall.

Kane, M. J., Brown, L. H., McVay, J. C., Silvia, P. J., Myin-Germeys, I., \& Kwapil, T. R. (2007). For whom the mind wanders, and when: An experience-sampling study of working memory and executive control in daily life. Psychological Science, 18, 614-621.

Kurzban, R., Duckworth, A., Kable, J., Myers, J. (2013) An opportunity cost model of subjective effort and task performance. Behavioral and Brain Sciences, 36, 661-79.

Liu, G., \& Liang, K. Y. (1997). Sample size calculations for studies with correlated observations. Biometrics, 53, 937-947.

Ma, Y., Mazumdar, M., \& Memtsoudis, S. G. (2013). Beyond repeatedmeasures analysis of variance: advanced statistical methods for the analysis of longitudinal data in anesthesia research. Regional Anesthesia and Pain Medicine, 37, 99-105. doi:10.1097/AAP.0 b013e31823ebc74

Marcora, S. M. (2010). Counterpoint: Afferent feedback from fatigued locomotor muscles is not an important determinant of endurance exercise performance. Journal of Applied Physiology, 108, 454 456.

Marcora, S. M., Staiano, W., \& Manning, V. (2009). Mental fatigue impairs physical performance in humans. Journal of Applied Physiology, 106, 857-864.

Muraven, M., \& Baumeister, R. F. (2000). Self-regulation and depletion of limited resources: Does self-control resemble a muscle? Psychological Bulletin, 126, 247-259. doi:10.1037/00332909.126.2.247

Muthalib, M., Anwar, A. R., Perrey, S., Dat, M., Galka, A., Wolff, S., ... Muthuraman, M. (2013). Multimodal integration of fNIRS, fMRI and EEG neuroimaging. Clinical Neurophysiology, 124, 20602062.

Nybo, L., \& Secher, N. H. (2004). Cerebral perturbations provoked by prolonged exercise. Progress in Neurobiology, 72, 223-261.

Obrig, H., \& Villringer, A. (2003). Beyond the visible-Imaging the human brain with light. Journal of Cerebral Blood Flow and Metabolism, 23, 1-18.

Paas, F. G., \& Van Merrienboer, J. (1993). The efficiency of instructional conditions: An approach to combine mental effort and performance measures. Human Factors, 35, 737-743.

Pardo, J. V., Fox, P. T., \& Raichle, M. (1991). Localization of a human system for sustained attention by positron emission tomography. Nature, 349, 61-64.

Perrey, S. (2008). Non-invasive NIR spectroscopy of human brain function during exercise. Methods, 45, 289-299. doi:10.1016/j. ymeth.2008.04.005

Pesce, C., Capranica, L., Tessitore, A., \& Figura, F. (2003). Focusing of visual attention under submaximal physical load. International Journal of Sport and Exercise Psychology, 1, 275-292.
Quaresima, V., Bisconti, S., \& Ferrari, M. (2012). A brief review on the use of functional near-infrared spectroscopy (fNIRS) for language imaging studies in human newborns and adults. Brain and Language, 121, 79-89.

Raichle, M. E., MacLeod, A. M., Snyder, A. Z., Powers, W. J., Gusnard, D. A., \& Shulman, G. L. (2001). A default mode of brain function. Proceedings of the National Academy of Sciences, 98, 676-682. doi:10.1073/pnas.98.2.676

Rejeski, W. J. (1985). Perceived exertion - An active or passive process? Journal of Sport Psychology, 7, 371-378.

Rooks, C. R., Thom, N. J., McCully, K. K., \& Dishman, R. K. (2010). Effects of incremental exercise on cerebral oxygenation measured by near-infrared spectroscopy: A systematic review. Progress in Neurobiology, 92, 134-150.

Rupp, T., \& Perrey, S. (2008). Prefrontal cortex oxygenation and neuromuscular responses to exhaustive exercise. European Journal of Applied Physiology, 102, 153-163.

Schmit, C., Davranche, K., Easthope, C., Colson, S., Brisswalter, J., \& Radel, R. (2015). Pushing to the limits: The dynamics of cognitive control during exhausting exercise. Neuropsychologia, 68, 71-81.

Silvestrini, N., \& Gendolla, G. (2013). Automatic effort mobilization and the principle of resource conservation: One can only prime the possible and justified. Journal of Personality and Social Psychology, 104, 803-816.

Steinbrink, J., Villringer, A., Kempf, F., Haux, D., Boden, S., \& Obrig, H. (2006). Illuminating the BOLD signal: Combined fMRI-fNIRS studies. Magnetic Resonance Imaging, 24, 495-505. doi:10.1016 /j.mri.2005.12.034

Stevenson, H., Russell, P. N., \& Helton, W. S. (2011). Search asymmetry, sustained attention, and response inhibition. Brain and Cognition, 77, 215-222. doi:10.1016/j.bandc.2011.08.007

Strangman, G., Culver, J. P., Thompson, J. H., \& Boas, D. A. (2002). A quantitative comparison of simultaneous BOLD fMRI and NIRS recordings during functional brain activation. NeuroImage, 17, 719-731.

Strangman, G., Zhang, Q., \& Li, Z. (2014). Scalp and skull influence on near infrared photon propagation in the Colin 27 brain template. NeuroImage, 85, 136-149.

Tempest, G. D., Eston, R. G., \& Parfitt, G. (2014). Prefrontal cortex haemodynamics and affective responses during exercise: A multichannel near infrared spectroscopy study. PLoS ONE, 9, e95924. doi:10.1371/journal.pone.0095924

Tucker, R. (2009). The anticipatory regulation of performance: The physiological basis for pacing strategies and the development of a perception-based model for exercise performance. British Journal of Sports Medicine, 43, 392-400.

Wang, C. C., Chu, C. H., Chu, I. H., Chan, K. H., \& Chang, Y. K. (2013). Executive function during acute exercise: The role of exercise intensity. Journal of Sport and Exercise Psychology, 35, 358-367.

Yamanaka, K., Yamagata, B., Tomioka, H., Kawasaki, S., \& Mimura, M. (2010). Transcranial magnetic stimulation of the parietal cortex facilitates spatial working memory: Near-infrared spectroscopy study. Cerebral Cortex, 20, 1037-1045. doi:10.1093/cercor/bhp163

Yamashita, S., Iwai, K., Akimoto, T., Sugawara, J., \& Kono, I. (2006). Effects of music during exercise on RPE, heart rate and the autonomic nervous system. Journal of Sports Medicine and Physical Fitness, 46, 425-430.

Ye, J. C., Tak, S., Jang, K. E., Jung, J., \& Jang, J. (2009). NIRS-SPM: Statistical parametric mapping for near-infrared spectroscopy. NeuroImage, 44, 428-447. doi:10.1016/j.neuroimage.2008.08.036 